\title{
Research Trend Analysis on Smartphone Addiction
}

\author{
Bo-Ra Kim ${ }^{1}, \mathrm{Mi}-\mathrm{Na} \mathrm{Lee}^{2}$ \\ ${ }^{I}$ The Doctor's Course, Department of Counseling Therapy, Kwangshin University, South Korea, \\ bora4638@naver.com \\ ${ }^{2}$ Professor, Welfare Counseling Convergence Department, Kwangshin University, South Korea, \\ lmn4780@naver.com
}

Corresponding author: Mi-Na Lee

\begin{abstract}
This study analyzed the research trend of smartphone addiction, focusing on domestic theses. The data were gathered from domestic theses from 2011 to 2020, and the surveyed research from 2009 to 2020 with a total of 885 articles. The research questions examined the research trends by degree type related to smartphone addiction, year, subject, and by subject type. The collected data includes 790 theses for the master's degree and 95 theses for the doctoral degree. First, in terms of research trends by year, the highest number of papers published in 2015 was 149(16.8\%). Second, in theses on adolescents, $392(44.2 \%)$ was the highest. Third, in the research trend by subject type, individual factors were the highest with 224(25.4\%). The popularization of smartphones has caused various problems as well as convenience. It affects not only the physical aspect, but it also causes economic problems, psychopathological problems, and disrupts work and studies. The degree of its effects is very serious, thus, smartphone addiction is widespread. The results of this study is intended to provide useful information for the development of prevention programs in the field of mental health for prevention of smartphone addiction according to the content by year, subject, and subject type.
\end{abstract}

Keywords: Smartphone Addiction, Hyper Immersion, Popularization, Adolescence, Trend Analysis

\section{Introduction}

\subsection{Smartphone Addiction}

Smartphone addiction is not yet an official medical diagnosis because of insufficient scientific evidence. Nevertheless, the reason why smartphone addiction should be paid attention is that there are people suffering from smartphone addiction in our society. There are many phenomena around us that can provide evidences on the possibility of smartphone addiction.

For example, when you leave your smartphone at home or when the battery is low, you are often overwhelmed with anxiety. This trend reflects'nomo phobia', a new term that refers to a symptom of being afraid of the absence of a mobile phone. In addition, when the Internet is not available, you feel annoyed or frustrated, and if you do not check text messages every few minutes, you may be worried about smartphone addiction. Smartphone addiction in our society is not recognized as a serious problem like drug addiction or gambling addiction among people because it is not subject to the primary regulation of the law like drugs or gambling. However, the reason why smartphone addiction should not be overlooked is that it is similar to the process of becoming "addicted" to drugs, and gambling. For example, drug addiction stimulates the brain when a specific drug is administered, and the

Received: February 07, 2021; $1^{\text {st }}$ Review Result: March 25, 2021; $2^{\text {nd }}$ Review Result: May 11, 2021 Accepted: June 30, 2021 
neurotransmitter dopamine is secreted, leading to a feeling of pleasure. At this time, the brain circuit through which dopamine is transmitted develops only when stimulation by drug administration is continuously performed. However, as the brain circuit develops, you cannot feel the same pleasure as before. Therefore, in order to obtain the same pleasure as before, more drug administration is required than before, which in turn leads to addiction.

Smartphone addiction also leads to immersion and indulgence, increasing reliance on smart phone devices when people feel pleasure or extreme pleasure through smart phone devices such as the Internet or smart phones. Increased dependence can lead to psychological anxiety, anxiety, stress, and a lack of concentration or even depression when certain situations in which a smartphone device is not available can lead to an inability to properly perform one's duties or tasks. The high convenience, practicality, and entertainment of smart phone devices such as smart phones are closely related to dependence on smart phone devices. Considering this, anyone who uses a smartphone device may be a potential smartphone addict who can lead to smartphone addiction without knowing it. In particular, smartphone addiction is very widespread, from children and adolescents to college students and even adults who engage in social activities.

\subsection{Smartphone Addiction Trend}

The first research on smartphone addiction in Korea was published in 2009, and since then, more than 880 papers have been published in academic research information service degree papers as of 2020. In addition, there were seven papers published in 2011, and they have been rapidly increasing since 2013, with more than 100 papers published annually since 2014 . This shows that as the penetration rate of smartphones increases, research on smartphone addiction is also actively underway. Smartphone addiction papers can be found in domestic dissertations and academic journals. In terms of publishing journal, it is published in various academic journals such as the Korea Digital Policy Association, the Korean Society of Science and Technology, the Korean Society of Children's Education, the Korea Rehabilitation Psychology, the Korea Youth Counseling and Welfare Institute, and the Korea Content Association. This can be said that efforts are ongoing to recognize and solve the problem of smartphone addiction in various fields.

Addiction is a habit-based addiction, which refers to a condition in which psychological and physical dependence prevents one from stopping an action and harms one's physical and mental health. There is a psychological dependence given the different meanings of addiction. Psychological dependence is a concept such as habituation that tries to relieve tension and emotional discomfort by continuing to use any stimulus. Recently, the concept of addiction has been defined as a "behavioral addiction" concept that goes beyond drug addiction or substance addiction to gambling, the Internet, games, and smartphones.

Global attention is focused on new behavioral addiction due to fast-developing media such as the Internet and smartphones related to the IT high-tech industry. It is true that the rapid spread of smartphones with the entry of the media information generation is causing serious problems with behavioral addiction in vulnerable groups, including teenagers. In particular, a recent survey of teenagers to those in their $20 \mathrm{~s}$, the main users of smartphones, showed that more than $10 \%$ of users are overusing smartphones, which is the seriousness of the problem. The preceding research conducted on children and adolescents stated that it is a continuation of smartphone and Internet addiction concepts and has a commonality in terms of users' psychological responses[1]. They also analyze the concept of smartphone addiction, as terms such as 'smartphone overuse', 'addiction tendency' and 'overindulgence' are not precisely defined to express smartphone addiction and are used or divided based on the degree of addiction[1].

Addiction is too absorbed in smartphones to concentrate on other things and not get out of them. It is 
likely that they become part of the "Smombie" tribe, a term that refers to those who cannot take their eyes off their smartphones while walking on the street[2]. People who are addicted to smartphones are likely to look into their smartphones even when they do other daily activities, and the risk of accidents increases. Professor Min Kyung-bok of Seoul National University's School of Preventive Medicine and a research team from Seoul National University's Institute of Health and Environment surveyed 608 college students, and $36.5 \%$ said they were addicted to smartphones.

In particular, students addicted to smartphones were 1.9 times more at risk of experiencing accidents such as slipping, colliding, and jammed subway doors during their daily lives than those who did not. The risk of falling or slipping was 2.8 times higher than the risk of collision, which was 1.83 times higher. Honolulu City, Hawaii, has implemented restrictions on smartphone use.

Addiction requires stronger intensity or duration stimulation to experience satisfaction. Smartphone tolerance is a state in which smartphone usage must be increased to have the same effect as before, and withdrawal means experiencing extreme mood swings, nausea, headaches, and physiological reactions such as insomnia when smartphone use is stopped or suddenly decreased. Conflict refers to the problem of excessive smartphone use causing conflicts with important daily activities such as interpersonal relationships, academic, social activities, and leisure activities. Relapse is the tendency to temporarily stop using smartphones or to revert back to the initial pattern even after having control. In other words, smartphone addiction is a state of continuous exploitation even though we know that excessive immersion in smartphone use will result in loss of control over it, resulting in negative consequences for ourselves and our surroundings[2]. Smartphone addiction is also closely related to mental health. If exposed to strong blue light from smartphones for a long time, there is a high risk of breaking the biorhythm. The body recognizes the day and night through light coming into the eyes and controls the rhythm of the body. Excessive use of smartphones during the night is easy for the human body to confuse day and night, raising the possibility of sleeping disorders and leading to mental illnesses such as depression and anxiety.

One out of every five smartphone users in Korea is at risk of overreliance, which is likely to suffer from problems in their daily lives due to excessive use of smartphones. In particular, caution is required because about one in every three teenagers is at risk of overreliance. According to the 2019 Smartphone Overdependence Survey released by the Ministry of Science and ICT with the Korea Information Society Agency, the proportion of Korean smartphone users who are at risk of overreliance increased by $0.9 \%$ to $20 \%$. Among the age groups, the proportion of children aged 3 to 9 years old who are at risk of overreliance on smartphones $2.2 \%$ to $22.9 \%$ during the same period. In particular, $30.2 \%$ of teenagers aged 10 to 19 are at risk of overreliance. According to the report, $18.8 \%$ of adults (20-59) and $14.9 \%$ of senior citizens in their 60 s are relatively dependent on smartphones among teenagers. infants and children (Seoul Economy, 2020).

This is linked to the quality of life throughout the life of children and adolescents, and has a lot to do with social problems. According to the World Health Organization, 20\% of teenagers worldwide are reported to experience mental health problems every year[3][4]. According to a study of the relationship between smartphone addiction and depression among 1,600 teenagers, smartphone addiction has a direct impact on suicidal thoughts and negative effects on self-habit and behavioral control[5].

In particular, the results of an in-depth interview with college students who think they are addicted to smartphones are as follows[6]. The use of smartphones was divided into five main categories. They are "struggling for relationships and communication," "overuse and loss of control," "psychological maladjustment," "threatening daily life," and "a glimpse of the possibility of recovery." A classified group of topics was analyzed as causality, which called 'struggle for relationship and communication' the cause of smartphone addiction[6]. The reason is that the desire to build relationships and communicate using smartphones is suggested.

According to the classification criteria in the previous chapter, the above paper has a view of finding 
the cause of smartphone addiction in the user's environment. In addition, 608 college students analyzed the four factors that diagnose smartphone addiction (daily life disorder, virtual world-oriented, withdrawal, immunity) and psychological anxiety and subjective health, investigating smartphone overuse, depression, anxiety, suicidal, thoughts and subjective health[7].

Smartphone addiction poses a serious negative effect and a significant threat to the mental health of teenagers. Studies show that smartphone addiction affects the mental health of teenagers[8][9]. As the usage rate of smartphones increases in adolescents, they are exposed to the risk of addiction. Nevertheless, teenagers should know why they use their smartphones too much. It can search for photos, information, and knowledge, and can vicariously satisfy the achievements and satisfactions that cannot be achieved in reality by listening to music, movies, and games in the virtual world, and communicate with many people easily and quickly through SNS and the Internet. Thus, adolescence is more aggressive toward people or animals than in childhood, and deviant behaviors such as destruction, fraud, or theft can occur repeatedly and continuously, showing explosive anger, low patience, instability, and rough behavior[10]. We looked at the effects of smartphone addiction trends and school violence experiences on mental health. First, anxiety and depression levels increase depending on teenagers' tendency to addiction to smartphones, and among them, it was most influential in daily life, and grades were related to the lower ranks. It is said that smartphone addiction directly affects suicidal thoughts and adversely affects self-habit, behavior, and control[5]. In other words, the stronger the tendency to addiction to smartphones, the higher the level of anxiety and depression, and depression is a common indicator of mental health and acts as a risk factor for increasing smartphone addiction. Mental health and school violence experiences caused by smartphone addiction in adolescence can cause more serious aftereffects than adults. Second, school violence experience is related to mental health. It is said that the school violence experience increases teenagers' aggression, anxiety, depression, and lower life satisfaction, and experiences low self-esteem, anxiety disorder, and depressive disorder after school violence[11][12].

Therefore, students who have experienced smartphone addiction trends and school violence are having an impact on mental health in their victimization and abuse experiences, so intervention in schools and at home in smartphone addiction and school violence should be urgently required[13]. It is a study that empirically reveals that teenagers' addiction to smartphones is not just about addiction, but is related to the pathological level of mental health, and it is necessary to come up with more specific measures for teenagers' mental health problems.

Recently, the term addiction has been avoided from this pathological point of view, and the general social phenomenon has led to a trend of focusing on prevention rather than treatment.

The Korea Information Society Agency decided to officially use the term smartphone overreliance rather than smartphone addiction[14]. Consequently, we define "the state in which excessive use of the Internet or smartphones increases the saliency of the Internet and reduces the control of uses, experiencing problematic consequences" as Internet overdependence[15]. This is similar to the concept of behavioral addiction, and the concepts of "addiction" and "existence" and "Internet" and "smartphones" have not yet been clearly distinguished, such as mixing the terms of Internet overreliance and smartphone overreliance. According to the 2017 National Informatization White Paper, the Internet usage rate using smartphones was $83.6 \%$, and those in their 20 s showed $99.7 \%$ of usage. The purpose of using the Internet was $91.6 \%$ for communication, such as chatting, SNS, and e-mail, followed by leisure activities, data and information acquisition, and applications such as communication (78.3\%), photography and video (62.2\%), news (54.4\%), games (22.7\%), and music (18.7\%). In mobile Internet service utilization, instant messenger, a communication tool, also topped the list, with mobile instant messenger utilization tallied at $100 \%$ for university students[14]. This form of smartphone communication is not only fast interaction, but also more hyper-connected society tendency than conventional conversational forms of text and voice calls[16]. 
Academic discussions on smartphone addiction are derived from research on Internet addiction and mobile phone addiction, and smartphone addiction is distinguished from Internet addiction and mobile phone addiction as the unique characteristics of smartphones are considered in the process. However, factors presented as classification criteria are concentrated on the mechanical properties of smartphones, which can hardly be considered to be distinguished by similar concepts or usage or content depending on the degree of characteristics. This causes confusion even in the use of terms related to smartphone addiction. Terms such as addiction, overuse, dependence, and overindulgence are being used without a clear distinction.

Teenagers' smartphone addiction is increasing over time. Activities to cope with this situation are centered on the "Internet Addiction Response Center (www.iapc.or.kr)" under the National Information Society Agency. The 'Internet Addiction Response Center' provides diagnostic tests on the website to determine whether Internet or smartphone addiction has occurred, and experts' addiction to the Internet and smartphones is conveniently used by phone, messenger, and online chat anywhere in the country. We are carrying out a consultation business where you can receive counseling, and to actively respond to addictions such as the Internet and smartphones, professional instructors are dispatched to schools and demanding institutions to provide preventive education. In addition, we are working on a business to nurture professional counselors who can respond to the surge in demand for professional counseling to respond to addiction to the Internet and smartphones. However, the response of the "Internet Addiction Response Center" deals with smartphone addiction as a sub-factor, centering on Internet addiction, so it is still insufficient to adequately respond to smartphone addiction. On the other hand, substantial socio-economic losses due to smartphone addiction and excessive use of smartphones are also large. Looking at this based on past studies on smartphones, the negative effects of smartphones on adolescents are exposure to electromagnetic waves when using smartphones for a long time, and electromagnetic waves damage important brain cells, causing problems such as headaches and decreased concentration. Can cause health problems.

Electromagnetic waves are most often generated at the antenna and the smartphone connection while waiting after dialing the phone number of the other party for a call, and it has already been scientifically proven and studied that it causes various diseases and even sleep disorders. In addition, there is an economic problem in which teenagers are charged a fee for using smartphones, which is difficult for adolescents to bear, by making calls with friends for a long time, sending excessive amounts of text messages, and using paid content. This problem causes conflict with parents, destroys the consumption life of adolescents, and results in the need to raise or earn money for adolescents who need to be faithful to school. In other words, it is reported that the biggest problem due to the use of smartphones in adolescence is a side effect of lifestyle changes. In other words, if you don't have a smartphone for a while, you feel anxious and empty, or after you have a smartphone, your homecoming time is delayed and the number of times you go out increases. I am holding on to what I am doing. In addition, teenagers with a total daily call volume between 20 and 30 minutes using a smartphone perceive the problem of using smartphones higher than those with less than 5 minutes, and adolescents who are worried about the charges for using smartphones may have conflicts with their parents. It was revealed that they perceived the problems caused by the use of smartphones higher than that of teenagers who are troubled by the teacher's interference, and said that the degree of perception of problems with mobile phones varies according to the level of use of mobile phones and the burden of usage charges. As such, the types of losses resulting from the use of smartphones are diverse and can have a negative impact on daily life. However, it can be seen that many adolescents show a tendency to be addicted to smartphones, but they use them without being aware of them, and they become increasingly dependent on smartphones without their knowledge and face various problems.

Through this study, we would like to analyze and investigate research trends on smartphone addiction based on domestic degree papers that have been published so far, and propose useful information to 
research on smartphone addiction. In this study, the analysis data on smartphone addiction research trends were analyze based on the degree papers published in Korea from 2009 to 2020, and the research problems are as follow. First, what is the degree type and annual distribution of papers related to smartphone addiction?

Second, what are the characteristics of the subject-specific research fields of papers related to smartphone addiction?

Third, what are the characteristics of subject-specific types of papers related to smartphone addiction?

\section{Research Method}

\subsection{Analysis Target}

This study analyzed domestic master's and doctorate papers on smartphone addiction in the National Assembly Library, riss.kr, KISS, and National Library of Korea. The core words used in the collection process were collected using smartphones, smartphone addiction, smartphone immersion, and smartphone addiction programs. The collected master's degree papers were 790 and $\mathrm{PhD}$ papers were 95 , a total of 885 papers.

\subsection{Data Processing}

In this study, 885 smartphone addiction-related dissertations from 2009 to 2020 were used in the analysis. In order to understand general trends, data were analyzed and processed by degree type, research trends by years, subject, and research subject by statistical method. In addition, we treat smartphone addiction-related research papers as data using Excel to analyze them in accordance with the element-specific analysis baselines. The analysis results were frequently analyzed by mistake and percentage, and the percentage of all analysis tables was rounded to the first place.

\section{Analysis Result}

\subsection{Annual Research Trends on Smartphone Addiction by Degree Type}

The annual research trends for each degree type on smartphone addiction was analyzed. When looking at the degree thesis and year-by-year on smartphone addiction, it is presented as a percentage separated by degree type and year from 2009 to 2020 , as shown in [Table 1].

[Table 1] Classification by Type and Year of Degree related to Smart Addiction.

\begin{tabular}{c|c|c|c|c|c|c|c|c|c|c|c|c|c|c}
\hline Sortation & 09 & 10 & 11 & 12 & 13 & 14 & 15 & 16 & 17 & 18 & 19 & 20 & Total & $(\%)$ \\
\hline Master & 1 & 0 & 6 & 27 & 77 & 134 & 137 & 115 & 89 & 78 & 72 & 54 & 790 & 89.4 \\
Doctor & 0 & 0 & 1 & 2 & 2 & 8 & 12 & 13 & 17 & 17 & 8 & 15 & 95 & 10.6 \\
Total & 1 & 0 & 7 & 29 & 79 & 142 & 149 & 128 & 106 & 95 & 80 & 69 & 885 & 100.0 \\
\hline
\end{tabular}

The collected analysis collected a research paper on smartphone addiction published from 2009 to 2020. A total of 885 papers were surveyed with 790 Master's Theses (89.4\%) and 95 Doctor's Theses (10.6\%), and the annual analysis showed that 149 (16.8\%) were published in 2015. 


\subsection{In Research on Smartphone Addiction by Subject}

Research trends for each subject of the degree paper on smartphone addiction were analyzed. Trends for each subject of research on smartphone addiction may be presented as shown in [Table 2].

[Table 2] The Smartphone Addiction Research Topics

\begin{tabular}{|c|c|c|c|}
\hline Sortation & Master's degree thesis & Doctor's degree thesis & Rate\%) \\
\hline $\begin{array}{c}\text { Toddler } \\
\text { Child } \\
\text { Teenage } \\
\text { University student } \\
\text { Adult } \\
\text { Parents and children } \\
\text { Others }\end{array}$ & $\begin{array}{c}35 \\
95 \\
356 \\
18 \\
83 \\
55 \\
147\end{array}$ & $\begin{array}{c}6 \\
15 \\
36 \\
17 \\
4 \\
3 \\
14\end{array}$ & $\begin{array}{c}41(4.6) \\
110(12.5) \\
392(44.2) \\
35(3.9) \\
87(10.0) \\
58(6.5) \\
161(18.2)\end{array}$ \\
\hline Total & 790 & 95 & $885(100.0)$ \\
\hline
\end{tabular}

As shown in [Table 2], research and analysis results on smartphone addiction were $392(44.2 \%)$ and the highest in thesis for teenagers, followed by 161 (18.2\%) for others and $110(12.5 \%)$ for children.

\subsection{The smartphone addiction research topics}

The researchers analyzed the trends by subject of research on smartphone addiction. When classifying research trends by types with research topics, they were classified into eight topics: individual factors, family factors, school factors, social factors, mental health areas, career areas, prevention areas, and others.

[Table 3] Comparative Analysis of Research Trends on Smartphone Addiction by Subject

\begin{tabular}{|c|c|c|c|}
\hline \multirow{2}{*}{ Sortation } & \multicolumn{2}{|c|}{ Number of thesis } & \multirow{2}{*}{ Rate (\%) } \\
\hline & Master & Doctor & \\
\hline Personal factors & 206 & 18 & $224(25.4)$ \\
\hline Family factor & 166 & 16 & $182(20.5)$ \\
\hline School factor & 92 & 5 & $97(10.9)$ \\
\hline Social factors & 121 & 31 & $152(17.1)$ \\
\hline The field of mental health & 69 & 7 & $76(8.5)$ \\
\hline Career area & 4 & 0 & $4(0.4)$ \\
\hline Prevention field & 20 & 6 & $26(2.9)$ \\
\hline Others & 120 & 4 & $124(13.9)$ \\
\hline Total & 798 & 87 & $885(100.0)$ \\
\hline
\end{tabular}

As shown in [Table 3], research trends on smartphone addiction were the highest among subject types with 224 (25.4\%), followed by family factors with 182 (20.5\%), social factors with $152(17.1 \%)$, followed by other, school factors, prevention, and career areas. 


\section{Conclusion}

It is not easy to cure addiction. This is because they are immersed in the long habit of addiction, the control of the frontal lobe executive function is reduced, and the habitual and automatic behavioral pattern of surrendering to the overlying level of impulsiveness or compulsion is developed. That's why it is difficult to set and implement long-term, high-level goals that are useful to a person. Therefore, it is very important in the first step of treatment to motivate to aim for a more balanced and higher level of life goal, rather than momentarily immersed in the target of addiction.

There will be no easy and simple solution that can be well controlled while properly restraining the targets or activities that are likely to be delicious and indulge and obsess over. First, awareness and awareness of the dangers of addiction problems, second, acquisition of self-regulation for fulfilling impulsive and self-centered needs, and third, development of social and moral interests should all be included[17].

Media addiction has emerged as one of the negative aspects as it enters the media age. In particular, in Korea, where media has developed rapidly, media addiction has also emerged quickly and strongly, becoming one of the serious addictions. In particular, it is very remarkable that adolescents are the main target among various addictions. Korea is one of the world's top countries in the use of various media, high-speed Internet infrastructure, and the spread of smartphones. Smartphone addiction is affected by personal psychological characteristics, the characteristics of the media itself, the characteristics of the contents produced by the media, and the environmental factors of the media users. In particular, in the process of using individual media and the personal characteristics of media users, the motivation and purpose of using the media users are combined with each other, causing a synergistic effect, leading to a more severe state of smartphone addiction.

The popularization of smartphones has caused many problems as well as convenience. Smartphone addiction, which can cause not only physical effects, but also economic problems, psychopathological problems, and disrupt work and study, is getting serious. Particularly noteworthy is that the smartphone addiction rate among teenagers is higher than that of adults, and the smartphone addiction rate is higher than the Internet addiction rate. Prior to the spread and popularization of smartphones, there were many studies on Internet and mobile phone addiction, and some recent research on smartphone addiction among teenagers focuses mainly on the effects of smartphone addiction on teenagers. Despite the rising smartphone addiction rate among teenagers and many problems, research on various factors that can affect teenagers' smartphone addiction and approach from an ecological perspective have to be made.

Studies of ecological system variables affecting smartphone addiction showed correlations between personal system factors, aggression, depression, self-control, parental parenting, parent-child communication, and parent-child addiction. Analysis of group-specific differences by smartphone addiction showed significant differences in aggression, depression, self-control, and parent-child communication (father) in family system factors[18].

Qualitative research among prior studies has derived various meanings of individuals' experiences in using smartphones, and the key to penetrating most studies is that interpersonal relationships are closely related to smartphone use experiences. According to communication theory, during "communication with others," "direct communication", The concept of "communication with others" is corresponding to interpersonal problems related to smartphone use experiences revealed by qualitative research. The concept of 'direct communication' seems to correspond to qualitative research on using smartphones due to interpersonal problems[19] as we define 'direct communication' as face-to-face communication.

Both sides of smartphone are also being revealed in terms of interpersonal relationships. In this regard, the concept of "direct communication" in humanities therapy has been applied, enabling discussions on what role smartphones can play in terms of communication with others. It is a person, not a tool, that is stated as an object of 'direct communication'. Using smartphones due to frustration in communication 
with others should eventually lead to 'direct communication' with others. Communication using smartphones can be considered as need to use it. However, using smartphones is causing problems because they are not able to meet the demands that correspond with them. On this issue, it can be seen in the discussion that "acceptance will" and "expression ability" determine the quality of communication through face-to-face contact with others. If the use of a smartphone functions in a way that improves the willingness to accept others and the ability to express oneself, it can be seen as a smartphone. However, according to prior research, smartphone users are experiencing interpersonal problems due to the use of smartphones, so the view of communication theory on this problem is likely to be valid.

Therefore, first, psychological support from adolescents is needed. An efficient program must be prepared and proceeded. Adolescents who are tired of academic stress, etc., need to grasp each individual's interests and areas of interest, form a group, and develop and operate programs tailored to their own characteristics. Accordingly, the school should provide an active support plan for experts to operate the program more effectively through systematic intervention. Therefore, education should be provided so that administrators, parents, and teachers of each school can correctly recognize the problem of smartphone addiction.

Second, it is self-inspection on the use of smartphones by adolescents. In schools, if meditation is practiced before class starts or during lunch time so that teenagers can control their smartphone use, self-concept and control ability will also improve if they self-inspect their smartphone usage habits and reduce smartphone usage time. I will be able to. In addition, a system that can provide various information related to smartphones to parents should be prepared to reduce the visual gap in the use of smartphones by adolescents. Third, it is the intervention of family counseling. Considering that problems such as smartphone addiction are caused by dysfunctional family problems, parents as well as adolescents should receive systematic family counseling together. To this end, health and family support centers, community welfare centers, mental health centers, and schools should allocate professional counselors and provide counseling for individuals and families so that in-person counseling or visiting counseling can be conducted at any time. In addition, educational programs should be developed and operated to strengthen the professionalism of counseling personnel.

Based on the limitations of this study, the direction of subsequent studies can be presented. Studies shall be conducted to collect and analyze data conducted on the subject of smartphone addiction. For a clearer conceptual arrangement, the study of the relevant concepts will need to be included. To establish a clear concept of smartphone addiction, research should be conducted in parallel with theoretical approaches. Since it should eventually lead to solving addiction problems, practical application research is also reasonable in the direction of follow-up research.

\section{References}

[1] M. J. Park, K. W. Kim, A Review on the Concepts within and Individual Psychological Variables and Clinical Intervention available for Smart Phone Addiction among Korean Children and Youth: Focusing on Studies Published in Korea from 2011 to 2015, Korean Journal of Play Therapy, (2015), Vol.18, No.2, pp.97-113, DOI : 10.17641/KAPT.18.2.2

[2] G. Y. Cho, Y. H. Kim, Factors Affecting Smartphone Addiction among University Students, Journal of the Korea Academia-Industrial cooperation Society, (2014), Vol.15, No.3, pp.1632-1640, https://doi.org/10.5762/KAIS.2014.15.3.1632

[3] World health statistics 2012, World Health Organization, https://apps.who.int/iris/handle/10665/44844

[4] S. J. Lee, A causal model between self-determination motivation, self-directed learning ability, learning commitment, and school happiness of middle school students participating in school sports clubs, Kyungnam University graduate school, Doctoral dissertation, (2018) 
[5] J. Y. Kim, H. J. Hwang, The mediating effects of self-control on the relationship between smartphone addiction and suicidal ideation in adolescents, Studies on Korean Youth, (2015), Vol.26, No.4, pp.59-84, http://dx.doi.org/10.14816/sky.2015.26.4.59

[6] K. S. Ko, M. J. Lee, Y. E. Kim, A Research on Addictive Use of Smartphone by University Students, Journal of Digital Contents Society, (2012), Vol.13. No 4, pp.501-516, https://doi.org/10.9728/dcs.2012.13.4.501

[7] Smartphone overuse data, (2017), www. kgnews. co. kr.

[8] I. S. Kang, The relationship between smartphone addiction and mental health in elementary school students, Kongju National University Graduate School of Education and Information, Master's Thesis, (2015)

[9] M. S. Park, The effect of smartphone addiction and academic stress on the mental health and self-esteem of high school students, Soonchunhyang University graduate school, Master's thesis, (2013)

[10] W. G. Kirk, Adolescent suicidal: A school based approach to assessment and intervene champaign, IL; Research Press, (1993)

[11] J. G. Park, A study on the effects of school violence on the emotions of children and adolescents and the moderating effect of social capital, Sungkyunkwan University, Doctoral dissertation, (2015)

[12] J. Y. Park, The Effects of Adolescents' Experience of School Violence on Depression and Anxiety, Jeju National University, Master's thesis, (2017)

[13] S. Y. Lee, M. N. Lee, The Effect of Youth's Smartphone Addiction Tendency and School Violence Experience on Mental Health, Journal of Character Education and Research, (2020), Vol.5, No.1, pp.51-71, http://dx.doi.org/10.46227/JCER.5.1.4

[14] National Informatization White Paper, (2017), http://www.nia.or.kr.

[15] National Informatization White Paper, (2018), http://www.nia.or.kr.

[16] H. G. Kim, Integrated analysis of smartphone use and addiction process: focusing on the reconceptualization of addiction, Korea University Graduate School, Doctoral dissertation, (2017)

[17] K. H. Kim, A way to overcome addiction, the modern dungeon, Korean Journal of Health Psychology, (2007), Vol.12, No.4, pp677-693, DOI : 10.17315/kjhp.2007.12.4.001

[18] S. I. Hwang, A Study on Ecological System Variables Affecting Adolescents' Smartphone Addiction, Chosun University Graduate School of Policy, Master's Thesis, (2013)

[19] I. J. Kim, A Theory of Humanities Therapy based on the Communication Model, Human and cultural research, (2010), Vol.25, pp.347-367. 